\title{
Inercia presupuestal y padecimientos crónico-degenerativos en México: una peligrosa combinación
}

\author{
Gabriela Tapia y Jaime Hernández \\ Universidad Autónoma de la Ciudad de México
}

\section{Resumen}

La investigación aborda desde una perspectiva de política pública el tema del presupuesto por programas en materia de salud pública federal en México en el periodo 2010-2015. Específicamente, analiza el Presupuesto de Egresos de la Federación (PEF) y los Ramos y programas cuyo objetivo es velar por la salud: Ramo 12, Secretaría de Salud; Ramo 50, IMSS; y Ramo 51, ISSSTE. El argumento del artículo es que el diseño presupuestal de la política de salud en México no responde, por lo menos presupuestalmente, a las necesidades epidemiológicas más apremiantes de los mexicanos: -la prevención de enfermedades crónico-degenerativas-, sino a una asignación inercial, que al no tomar en cuenta estas prioridades y sus proyecciones demográficas vinculadas, pone en riesgo el sostenimiento del sistema de salud a futuro. La investigación tiene dos bases metodológicas: la primera, la identificación de la inercia de los presupuestos del sector salud. Lo anterior vía la comparación de los presupuestos de los Ramos que componen el PEF entre 2010 y 2015 (40 Ramos) y los Ramos destinados específicamente al sector salud (3 Ramos). La segunda, el análisis de política pública como proceso de solución de problemas e hipótesis causal (Aguilar, 2010: 32). El objeto de esta segunda aproximación es observar la relación causal entre la prevención de los principales problemas epidemiológicos en México y la distribución del presupuesto vía los temas de los programas aprobados en el PEF entre 2010 y 2015 en los Ramos del sector salud.

Palabras clave: Salud pública, inercia presupuestal, incrementalismo, política pública, prevención, presupuesto, programas presupuestarios, México.

Budget inertia and cronic degenerative diseases in Mexico: a dangerous combo

\begin{abstract}
This research studies budget allocated to health programs in Mexico during 2010-2015. Mainly, it studies Federal Budget Number 12 (Ministry of Health), Number 50 (Ministry of Social Securit/IMSS) and Number 51 (ISSSTE). Specifically, the ammount of money assigned to programs on those budgets whose main purpose is to look after public health in Mexicans. The main argument of the paper is that budget design on health issues does not respond to epidemiological needs -prevention of cronic degenerative diseases- and follows an inertial pattern of allocation that may prove dangerous for the sustainability of the mexican health system in the future. The reserach has two methodological basis: the first one, the identification of inertia or incrementalism on health budgets. This is observed through new programs on health policy over the last five years and budget allocated. The second one, public policy analysis as a process of problem solution and causal hypothesis (Aguilar 2010:32). The purpose of this second approach is to observe the causal relation between the main epidemiological problems in Mexico and the distribution of the health budget during 2010-2015.
\end{abstract}

Keywords: Public health, budget inertia, incrementalism, public policy, prevention, budget, budget programs, Mexico. 


\section{Introducción}

La investigación aborda desde una perspectiva de política pública el tema del presupuesto por programas en materia de salud pública federal en México en el periodo 2010-2015. Específicamente, analiza el Presupuesto de Egresos de la Federación (PEF) y los Ramos y programas cuyo objetivo es velar por la salud pública: Ramo 12, Secretaría de Salud; Ramo 50, IMSS; y Ramo 51, ISSSTE. El argumento del artículo es que el diseño presupuestal de la política de salud en México pareciera ser más inercial, que una respuesta a las necesidades epidemiológicas más apremiantes de los mexicanos. Si lo fuera, en el presupuesto se observarían mayores recursos para programas cuyo énfasis está en la prevención de enfermedades crónico-degenerativas, situación que no se identificó.

El estudio se basa en dos aproximaciones metodológicas: La primera, la identificación de la inercia del presupuesto del sector salud vía la comparación de las asignaciones de los Ramos que componen el PEF (43) con los Ramos destinados específicamente a la atención de la salud (Ramo 12, 50 y 51). La segunda, el análisis de política pública como proceso de solución de problemas e hipótesis causal (Aguilar, 2010: 32). El objeto de esta segunda aproximación es observar o no, la relación causal entre los principales problemas epidemiológicos en México actualmente los problemas crónico degenerativos-, y su presencia o no vía programas para su prevención en el PEF entre 2010 y 2015.

Como se mencionó, la primera aproximación metodológica consiste en identificar la inercialidad de la presupuestación en el sector salud. La inercia presupuestal parte de lo que en políticas públicas se conoce como incrementalismo. El concepto de incrementalismo sugiere que las decisiones públicas siguen la regla pragmática de salir del atolladero, del lío, reduciendo el universo de las opciones de política a ponderar, limitando las comparaciones sólo al conjunto de las políticas que difieren muy poco de las políticas que se llevarán actualmente a cabo y estudiando sólo los aspectos en que la opción propuesta y sus consecuencias difieren del statu quo (Aguilar, 1992: 48). En materia presupuestal, esto quiere decir que los presupuestos son similares año con año, sin tomar en cuenta aspectos importantes como cambios en las necesidades a atender o resultados de evaluaciones a los programas. En el sentido de esta investigación, la inercialidad se observa mediante los cambios o no de los programas presupuestales en los últimos años así como las modificaciones a su presupuesto. El presupuesto no se califica como incremental si se observan cambios en los programas así como cambios en el gasto en los últimos cinco años. Por el contrario, si los programas permanecen año con año al igual que sus presupuestos, se califican las políticas como inerciales.

La segunda aproximación, el análisis de política pública como proceso de solución de problemas e hipótesis causal, parte de una situación social indeseable que se identifica como objeto de intervención gubernamental (Aguilar, 2010: 32). En el caso de esta investigación, la situación social indeseable se compone de los principales problemas epidemiológicos que existen actualmente en el país: las enfermedades crónico degenerativas. La propuesta es que la respuesta que podría disminuir en mayor medida el problema público identificado debería hallarse en el conjunto de los programas que integran los ramos presupuestales analizados, dado que el objetivo de éstos es velar por la salud pública. Así, se observa si existe o no relación causal entre las distintas situaciones sociales (epidemiológicas) indeseables y la acción gubernamental a través del presupuesto federal, concretamente, de los Ramos 12, 50 y 51.

El resultado de ambas aproximaciones metodológicas lleva a la conclusión de que la presupuestación en materia de salud en México es inercial, y que la prevención de los padecimientos crónico degenerativos, que debiera ser un aspecto sustancial de la estrategia en la política de salud pública en México está prácticamente ausente del Presupuesto de Egresos de la Federación o por lo menos, no se refleja en las temáticas de los programas de la Secretaría de Salud, IMSS e ISSSTE durante el periodo 2010-2015. Si se observara la hipótesis causal esperada y por tanto el gasto no fuera inercial, este se concentraría actualmente en la prevención de enfermedades crónico degenerativas. Temas como la nutrición, la prevención de la obesidad, la disminución en el consumo de cigarro y alcohol y la activación física deberían dominar las temáticas de los programas presupuestales. Desafortunadamente, esta situación no se encuentra en el análisis llevado a cabo.

El artículo está organizado en cuatro apartados. En el primero, se analizan los 43 Ramos del PEF y se compara su inercialidad contra la de los Ramos cuyo objetivo es velar por la salud pública. En el segundo apartado se analizan los principales problemas epidemiológicos en México, sus causales, proyecciones demográficas e implicaciones para el gasto en salud a futuro. En el tercer apartado, se analizan los Ramos 12, 50 y 51 vinculándolos con la prevención de las enfermedades crónico degenerativas. Finalmente, se presentan conclusiones al respecto. 


\section{Inercialidad y presupuestación en el sector salud}

El tema del incrementalismo en el presupuesto tiene sus antecedentes teóricos en el análisis para el diseño de política pública. En esta etapa del ciclo, existen dos corrientes principales y radicalmente contrastantes de análisis: el racionalismo y el incrementalismo (Aguilar, 1992). El primero de ellos, el paradigma dominante en la literatura de toma de decisiones, surge de los escritos de Lasswell en el periodo de la posguerra, y el segundo, asociado con la inercialidad del presupuesto y propuesta de Lindblom, es de acuerdo al autor el tipo de análisis más utilizado por los hacedores de política.

El análisis racional se caracteriza por incorporar explícitamente el modelo de decisión racional o de solución racional de problemas, cuyos pasos son: determinar el objetivo a lograr y ordenar las preferencias, indicar los opciones de acción que por lo medios que incorporan y el cálculo de sus consecuencias se consideran idóneas para efectuar el estado de cosas-objetivo de la acción, comparar las acciones en términos de eficiencia técnica y económica, elección y puesta en práctica programada de la alternativa eficiente u óptima, evaluación de resultados, aprendizaje reiterativo según los resultados (Aguilar, 1992: 45). Un diseño de presupuesto estrictamente racional y técnico, sería un presupuesto en el que la asignación anual a los programas considerara además de muchos otros aspectos, los resultados de los distintos tipos de evaluación llevadas a cabo a los programas que lo componen. A evaluaciones positivas, no se esperarían cambios a los bienes y servicios entregados y por tanto tampoco modificaciones importantes en las asignaciones presupuestales. En este tipo de situación, los programas tal cual están diseñados, estarían solucionando los problemas públicos identificados vía los productos entregados. A evaluaciones negativas sin embargo, se esperaría observar cambios en los programas presupuestales acordes con los resultados de las distintas evaluaciones y probablemente por tanto cambios en los bienes y servicios y en la asignación presupuestal.

Contrario al esquema de análisis racional está la reacción de Charles Lindblom (1959, 1963, 1979), quien amplía la tesis de racionalidad limitada de Simon y la de ingeniería social gradual de Popper, al afirmar que el modelo racional no describe la decisión y el comportamiento efectivo de los gobernantes de carne y hueso. De acuerdo al autor, las decisiones son más bien inerciales y siguen la regla pragmática de "salir del atolladero, del lío"... y dos son los rasgos importantes del procedimiento: "el primero, consiste en reducir el universo de las opciones de política a ponderar, limitando las comparaciones sólo al conjunto de las políticas que difieren muy poco de las políticas que se llevarán actualmente a cabo... y el segundo, estudiar sólo los aspectos en que la opción propuesta y sus consecuencias difieren del statu quo" (Aguilar, 1992: 48). En este sentido, es importante tener en cuenta que la propuesta de Lindblom refiere al análisis de toma de decisiones en general y no específicamente al tema presupuestal.

La incorporación del incrementalismo a la teoría sobre presupuesto es obra de Wyldavsky (1964, 1975) y Fenno. De acuerdo a Wyldavsky (1964) el incrementalismo es un proceso en el que las bases presupuestales (gastos previos) son aceptadas, y la toma de decisiones se concentra en el cambio desde la base (Berry, 1990: 171). En este sentido, un presupuesto con características incrementales o inerciales es un presupuesto muy similar año con año, con pequeñas modificaciones respecto al presupuesto anterior y en el que elementos de racionalidad tales como la incorporación de resultados de evaluación como parte de su rediseño no son significativas.

Como se mencionó, en este apartado, se presentó el análisis de los 43 Ramos del PEF con el objetivo de conocer qué tan inercial es el gasto de aquellos ramos cuyo objetivo es velar por la salud pública en México: Ramo 12, 50 y 52 (Secretaría de Salud, IMSS e ISSSTE respectivamente). En México, el Presupuesto de Egresos de la Federación se compone de programas y proyectos con enfoque de resultados e impactos alineados con el Plan Nacional de Desarrollo (PND). Se conoce como Estructura Programática (art. 2, fracción XXII, Ley Federal de Presupuesto y Responsabilidad Hacendaria (LFPRH)), al conjunto de categorías y elementos programáticos que definen las acciones que efectúan los ejecutores de gasto para alcanzar sus objetivos y metas de acuerdo con las políticas definidas en el PND y en los programas y presupuestos. La estructura programática ordena y clasifica las acciones de los ejecutores de gasto (los distintos Ramos) para delimitar la aplicación del gasto y permite conocer el rendimiento esperado de la utilización de los recursos públicos.

Los programas presupuestarios están clasificados por ramos y cada ramo está conformado por programas. Se conoce como Ramo al elemento que identifica y clasifica el gasto público federal por entidades administrativas del Poder Ejecutivo (Secretarías, Departamentos de Estado, Presidencia de la República, Procuraduría General de la República), Poder Legislativo, Judicial, Ramos Generales de Gastos, Empresas Productivas del Estado, Organismos Autónomos y Entidades de Control Direc- 
to. Esta investigación se concentra en 43 de ellos, paticularmente, los que contienen programas con objetivos específicos establecidos en reglas de operación y que pueden ser analizados debido a que cuentan con matrices de indicadores que permiten su monitoreo y evaluación ${ }^{1}$.

El Presupuesto de Egresos de la Federación existen ramos administrativos, que engloban las Secretarías, ramos generales como el Instituto Nacional Electoral (INE), ramos autónomos como el Instituto Nacional de Transparencia, Acceso a la Información y Protección de Datos Personales (INAI), empresas productivas del estado como Petróleos Mexicanos (PEMEX) y Comisión Federal de Electricidad (CFE) y entidades de control directo como el Instituto Mexicano del Seguro Social (IMSS) y el Instituto de Seguridad y Servicios Sociales de los Trabajadores del Estado (ISSSTE).

En el caso de la primera parte de esta investigación, el análisis se basa en la comparación de los programas y presupuestos de los siguientes Ramos:

- Ramo 12, Secretaría de Salud (Ramo Administrativo)

- Ramo 50, Instituto Mexicano del Seguro Social (Entidad de Control Directo)

- Ramo 51, Instituto de Seguridad y Servicios Sociales de los Trabajadores del Estado, ISSSTE (Entidad de Control Directo)

Para iniciar el análisis es importante aclarar, que los Ramos de Salud en México se encuentran en los primeros siete lugares de los 43 ramos respecto a presupuesto asignado en los últimos cinco años. El IMSS, se ubica en cuarto lugar y el ISSSTE en séptimo lugar.

En la tabla 2, se presenta información que brinda indicios sobre la inercia del presupuesto en México al mostrar el número de programas por Ramo por año de 2010 a 2014. Como se observa, por lo menos en número, no existen grandes variaciones. Incluso, existen Secretarías y Órganos, que tienen el mismo número de programas desde el 2010. Es de interés el caso de los Ramos destinados a la salud pública. En este caso, observamos que el Ramo 12, correspondiente a Secretaría de Salud ha tenido solo dos variaciones en programas en cinco años. El IMSS ninguna y el ISSSTE una.

Esta inercialidad sin embargo podría no ser problemática. Sobre todo si el gasto estuviera dirigi-

\footnotetext{
${ }^{1} \operatorname{Los} \operatorname{Ramos} 24,26,28,29,30,34$, y 49 no se analizaron debido a que son recursos que no tienen aplicación vía programas específicos. Por ejemplo pago de deuda y créditos fiscales.
}

do a la atención de la prevención de enfermedades crónico degenerativas por ejemplo a la reducción del consumo de alcohol, o a la atención de temas de nutrición, obesidad o tabaquismo. Sin embargo, como se presenta a detalle en la última sección, esto no es así.

En la Tabla 3 se contabilizan el número de programas nuevos por año por Ramo. Al analizar los ramos destinados a la atención de la salud en México se observa que el Ramo 12, correspondiente a la Secretaría de Salud, sólo registra 9 programas nuevos en los últimos cinco años, el ramo 50 correspondiente al IMSS sólo 1 y el Ramo 51 correspondiente al ISSSTE también sólo uno. Es importante mencionar que estos ramos son también, de acuerdo a las encuestas relacionadas con satisfacción por los servicios que ofrecen, los de puntaje más bajo de todos, menores al 50 por ciento (Székely, 2013: 8). La inercia del presupuesto en salud además, no empata con los grandes cambios que ha experimentado el perfil de demanda de población y sus necesidades de salud en los últimos años, aspecto que se abordará en la última sección del documento.

Desafortundamente, aunque los tomadores de decisión en materia de políticas de salud tienen actualmente herramientas diferentes que les permitirían contar con una visión más detallada sobre los problemas que afectan a hombres y mujeres, no se recurre a ellas para evaluar y generar evidencia en la adecuación y diseño de los programas de salud (Lozano et al., 2013: 592). Resultados como estos obligarían a pensar en rediseñar los programas en este sector, sin embargo, en México no se observa en general una relación importante entre resultados de evaluaciones, rediseño de políticas y en consecuencia, asignación presupuestal (Tapia Téllez, 2016: 13-14).

Como se ve en la Gráfica 2, prácticamente no ha habido cambios en los programas del sector salud en los últimos cinco años. En el caso del Ramo 12 (Secretaría de Salud), en 2010 se registraron 35 programas presupuestarios, mientras que en 2015 se registraron 37. En el caso del Ramo 50 (IMSS) se identificaron los mismos 18 programas entre 2010 y 2015. En el caso del ISSSTE en 2010 había 43 programas y en 2015 se identificaron 44. Lo anterior, además de reflejar una inercia presupuestal en términos de programas en materia de salud en los tres ramos destinados a procurarla, también representa un riesgo para las finanzas públicas al tomar en cuenta los cambios epidemiológicos que estudiaremos en la siguiente sección, asi como las proyecciones demográficas y los costos asociados a la misma.

Como se mencionó, la inercia presupuestal es uno 
Tabla 1: Número de programas 2010-2015 por Ramo

\begin{tabular}{|c|c|c|c|c|c|c|c|}
\hline Ramo & Nombre del Ramo & 2010 & 2011 & 2012 & 2013 & 2014 & 2015 \\
\hline 1 & Legislativo & 4 & 4 & 4 & 4 & 4 & 4 \\
\hline 2 & Presidencia de la República & 4 & 7 & 7 & 7 & 8 & 6 \\
\hline 3 & Poder Judicial & 1 & 1 & 1 & 1 & 1 & 1 \\
\hline 4 & Gobernación & 28 & 30 & 39 & 48 & 49 & 47 \\
\hline 5 & Relaciones Exteriores & 20 & 20 & 23 & 19 & 17 & 18 \\
\hline 6 & Hacienda y Crédito Público & 66 & 72 & 76 & 69 & 56 & 50 \\
\hline 7 & Defensa Nacional & 17 & 19 & 20 & 20 & 19 & 20 \\
\hline 8 & $\begin{array}{l}\text { Agricultura, Ganadería, Desarrollo Rural, Pesca y } \\
\text { Alimentación }\end{array}$ & 36 & 39 & 38 & 39 & 39 & 36 \\
\hline 9 & Comunicaciones y Transportes & 47 & 46 & 50 & 48 & 45 & 40 \\
\hline 10 & Economía & 48 & 44 & 42 & 40 & 34 & 31 \\
\hline 11 & Educación Pública & 153 & 145 & 120 & 111 & 60 & 59 \\
\hline 12 & Salud & 34 & 33 & 34 & 34 & 37 & 36 \\
\hline 13 & Marina & 13 & 14 & 14 & 14 & 15 & 15 \\
\hline 14 & Trabajo y Previsión Social & 19 & 18 & 19 & 18 & 18 & 17 \\
\hline 15 & Desarrollo Agrario, Territorial y Urbano & 18 & 19 & 19 & 32 & 33 & 31 \\
\hline 16 & Medio Ambiente y recursos Naturales & 83 & 69 & 74 & 68 & 75 & 74 \\
\hline 17 & Procuraduría General de la República & 10 & 13 & 15 & 14 & 14 & 15 \\
\hline 18 & Energía & 25 & 23 & 26 & 27 & 27 & 22 \\
\hline 19 & Aportaciones a Seguridad Social & 22 & 21 & 21 & 21 & 22 & 21 \\
\hline 20 & Desarrollo Social & 34 & 36 & 36 & 31 & 29 & 31 \\
\hline 21 & Turismo & 16 & 18 & 22 & 17 & 18 & 19 \\
\hline 22 & Instituto Federal Electoral & 9 & 9 & 9 & 11 & 11 & 11 \\
\hline 23 & Provisiones Salariales y Económicas & 61 & 80 & 52 & 26 & 31 & 28 \\
\hline 25 & $\begin{array}{l}\text { Provisiones y Aportaciones para los Sistemas de } \\
\text { Educación Básica, Normal, Tecnológica y de Adul- } \\
\text { tos }\end{array}$ & 17 & 16 & 20 & 17 & 11 & 10 \\
\hline 27 & Función Pública & 11 & 11 & 11 & 11 & 12 & 11 \\
\hline 31 & Tribunales Agrarios & 4 & 4 & 4 & 4 & 4 & 4 \\
\hline 32 & Tribunal Federal de Justicia Fiscal y Administrativa & 3 & 3 & 3 & 3 & 5 & 3 \\
\hline 33 & $\begin{array}{l}\text { Aportaciones Federales para Entidades Federativas } \\
\text { y Municipios }\end{array}$ & 253 & 253 & 12 & 12 & 12 & 15 \\
\hline 35 & Comisión Nacional de los Derechos Humanos & 24 & 25 & 32 & 31 & 31 & 32 \\
\hline 36 & Antes Seguridad Pública & 14 & 12 & 11 & 0 & 0 & 0 \\
\hline 37 & Consejería Jurídica del Ejecutivo Federal & 3 & 3 & 3 & 3 & 3 & 3 \\
\hline 38 & Consejo Nacional de Ciencia y Tecnología & 18 & 16 & 17 & 16 & 18 & 18 \\
\hline 40 & Información Nacional Estadística y Geográfica & 6 & 6 & 5 & 6 & 6 & 6 \\
\hline 41 & Comisión Federal de Competencia Económica & 0 & 0 & 0 & 3 & 3 & 3 \\
\hline 42 & $\begin{array}{l}\text { Instituto Nacional para la Evaluación de la Educa- } \\
\text { ción }\end{array}$ & 0 & 0 & 0 & 3 & 7 & 7 \\
\hline 43 & Instituto Federal de Telecomunicaciones & 0 & 0 & 0 & 3 & 4 & 5 \\
\hline 44 & $\begin{array}{l}\text { Instituto Federal de Acceso a la Información y Pro- } \\
\text { tección de Datos }\end{array}$ & 0 & 0 & 0 & 0 & 7 & 7 \\
\hline 45 & Comisión Reguladora de Energía & 0 & 0 & 0 & 0 & 0 & 3 \\
\hline 46 & Comisión Nacional de Hidrocarburos & 0 & 0 & 0 & 0 & 0 & 4 \\
\hline \multicolumn{8}{|c|}{ Entidades de Control Directo } \\
\hline 47 & Petróleos Mexicanos & 21 & 21 & 21 & 22 & 22 & 22 \\
\hline 48 & Comisión Federal de Electricidad & 22 & 45 & 26 & 25 & 26 & 26 \\
\hline 50 & Instituto Mexicano del Seguro Social & 17 & 17 & 18 & 18 & 18 & 17 \\
\hline 51 & $\begin{array}{l}\text { Instituto de Seguridad y Servicios Sociales de los Tra- } \\
\text { bajadores del Estado }\end{array}$ & 44 & 45 & 44 & 45 & 44 & 45 \\
\hline
\end{tabular}

Fuente: Tapia Téllez, 2016: 10-11 
Tabla 2: Número de programas nuevos por año por Ramo

\begin{tabular}{|c|c|c|c|c|c|c|}
\hline Ramo & Nombre del Ramo & 2011 & 2012 & 2013 & 2014 & 2015 \\
\hline 1 & Legislativo & 0 & 1 & 0 & 0 & 0 \\
\hline 2 & Presidencia de la República & 3 & 0 & 0 & 1 & 0 \\
\hline 3 & Poder Judicial & 0 & 0 & 0 & 0 & 0 \\
\hline 4 & Gobernación & 2 & 9 & 13 & 7 & 0 \\
\hline 5 & Relaciones Exteriores & 0 & 4 & 0 & 1 & 0 \\
\hline 6 & Hacienda y Crédito Público & 8 & 10 & 2 & 6 & 1 \\
\hline 7 & Defensa Nacional & 2 & 5 & 0 & 0 & 1 \\
\hline 8 & Agricultura, Ganade ría, Desarrollo Rural, Pesca y Alimentación & 13 & 3 & 4 & 11 & 0 \\
\hline 9 & Comunicaciones y Transportes & 0 & 4 & 3 & 7 & 2 \\
\hline 10 & Economía & 0 & 0 & 5 & 1 & 0 \\
\hline 11 & Educación Pública & 14 & 17 & 4 & 14 & 0 \\
\hline 12 & Salud & 0 & 4 & 1 & 4 & 0 \\
\hline 13 & Marina & 1 & 0 & 0 & 1 & 0 \\
\hline 14 & Trabajo y Previsión Social & 0 & 1 & 0 & 1 & 0 \\
\hline 15 & Desarrollo Agrario, Territorial y Urbano & 1 & 2 & 15 & 4 & 0 \\
\hline 16 & Medio Ambiente y recursos Naturales & 9 & 13 & 0 & 10 & 1 \\
\hline 17 & Procuraduría General de la República & 4 & 2 & 0 & 1 & 0 \\
\hline 18 & Energía & 1 & 5 & 1 & 2 & 2 \\
\hline 19 & Aportaciones a Seguridad Social & 1 & 1 & 1 & 1 & 4 \\
\hline 20 & Desarrollo Social & 2 & 0 & 6 & 1 & 1 \\
\hline 21 & Turismo & 2 & 5 & 0 & 3 & 1 \\
\hline 22 & Instituto Federal Electoral & 0 & 0 & 6 & 0 & 0 \\
\hline 23 & Provisiones Salariales y Económicas & 25 & 20 & 5 & 8 & 17 \\
\hline 25 & $\begin{array}{l}\text { Provisiones y Aportaciones para los Sistemas de Educación Bási- } \\
\text { ca, Normal, Tecnológica y de Adultos }\end{array}$ & 5 & 12 & 0 & 3 & 2 \\
\hline 27 & Función Pública & 0 & 0 & 1 & 1 & 0 \\
\hline 31 & Tribunales Agrarios & 0 & 0 & 0 & 0 & 0 \\
\hline 32 & Tribunal Federal de Justicia Fiscal y Administrativa & 0 & 0 & 0 & 2 & 0 \\
\hline 33 & Aportaciones Federales para Entidades Federativas y Municipios & 0 & 12 & 0 & 0 & 4 \\
\hline 35 & Comisión Nacional de los Derechos Humanos & 1 & 7 & 0 & 0 & 0 \\
\hline 36 & Antes Seguridad Pública & 1 & 1 & 0 & 0 & 0 \\
\hline 37 & Consejería Jurídica del Ejecutivo Federal & 0 & 0 & 0 & 0 & 0 \\
\hline 38 & Consejo Nacional de Ciencia y Tecnología & 1 & 1 & 0 & 2 & 1 \\
\hline 40 & Información Nacional Estadística y Geográfica & 1 & 1 & 1 & 0 & 0 \\
\hline 41 & Comisión Federal de Competencia Económica & 0 & 0 & 3 & 0 & 0 \\
\hline 42 & Instituto Nacional para la Evaluación de la Educación & 0 & 0 & 3 & 4 & 2 \\
\hline 43 & Instituto Federal de Telecomunicaciones & 0 & 0 & 3 & 1 & 1 \\
\hline 44 & Instituto Federal de Acceso a la Información y Protección de Datos & 0 & 0 & 0 & 7 & 0 \\
\hline 45 & Comisión Reguladora de Energía & 0 & 0 & 0 & 0 & 3 \\
\hline 46 & Comisión Nacional de Hidrocarburos & 0 & 0 & 0 & 0 & 4 \\
\hline \multicolumn{7}{|c|}{ Entidades de Control Directo } \\
\hline 47 & Petróleos Mexicanos & 0 & 0 & 1 & 0 & 1 \\
\hline 48 & Comisión Federal de Electricidad & 5 & 2 & 0 & 1 & 0 \\
\hline 50 & Instituto Mexicano del Seguro Social & 0 & 1 & 0 & 0 & 0 \\
\hline 51 & $\begin{array}{l}\text { Instituto de Seguridad y Servicios Sociales de los Trabajadores del } \\
\text { Estado }\end{array}$ & 1 & 0 & 0 & 0 & 0 \\
\hline
\end{tabular}

Fuente: Tapia Téllez, 2016: 13-14 
Figura 1: Número de programas de Ramos de Salud 2010 y 2015

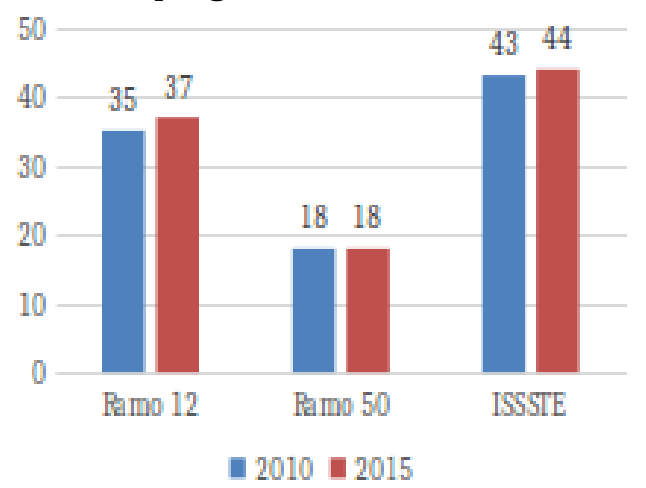

Fuente: elaboración propia con base en información del PEF 2010-2015

de los elementos que dan cuenta de una tendencia incremental en el diseño de una política pública. Sin embargo, la perspectiva incremental en una política funciona en países en los que las cosas "van bien", en los que las políticas públicas están dando resultados eficientes, pero no, en aquellos en los que la bola de nieve sólo se va haciendo más grande (Dror), como es el caso de México de los problemas epidemiológicos. En el sector salud por tanto, no se debería observar una presupuestación inercial.

\section{Problemas epidemiológicos en México y proyecciones de- mográficas}

El objetivo de esta sección es la identificación de los principales problemas epidemiológicos en México y sus proyecciones demográficas para posteriormente, en la siguiente sección, identificar si estos problemas y sus causales están siendo atendidos vía el análisis de los temas que abordan los programas presupuestarios de los Ramos 12, 50 y 51 (Secretaría de Salud, IMSS e ISSSTE respectivamente). El análisis de estos problemas se divide por grupo etario: niños, jóvenes, adultos y adultos mayores.

En niños, el primer problema epidemiológico identificado es la inseguridad alimentaria relacionada directamente con la desnutrición- (Instituto Nacional de Estadística Geografía, 2014b: 10). El problema de la inseguridad alimentaria es relevante, desde un punto de vista de política pública, si se tiene en cuenta que tres de cada cuatro hogares mexicanos en los que habitan menores de cinco años viven con este problema y uno de cada tres se encuentra en inseguridad alimentaria moderada o severa (Cuevas-Nasu et al., 2014: 552). Estudios recientes muestran un aumento del riesgo de desnutrición crónica. Ese riesgo es $42 \%$ mayor en menores de cinco años que viven en hogares con inseguridad alimentaria severa respecto de aquellos que viven en situación de seguridad alimentaria (Cuevas-Nasu et al., 2014: 552). Sobre este problema, es importante resaltar que hogares como los indígenas y de localidades rurales concentran las mayores prevalencias de inseguridad alimentaria moderada o severa y también es ahí donde se concentran las mayores proporciones de niños con desnutrición crónica (Cuevas-Nasu et al., 2014: 552).

La inseguridad alimentaria también se asocia a problemas como la anemia, en menores de dos años alcanza a casi 4 de cada 10 niños y en niños de 2 a 5 años a casi $20 \%$. Por otra parte, uno de cada cinco niños menores de cinco años que viven en hogares con estas características, presenta sobrepeso $(9.7 \%)$. En los hogares con inseguridad alimentaria moderada y severa habitan hasta $30 \%$ de escolares con sobrepeso y obesidad (Shamah-Levy et al., 2014: 582). La obesidad se genera debido a que la población en pobreza, satisface sus necesidades de consumo de calorías adquiriendo alimentos de bajo costo, altos en energía pero con un contenido deficiente de micronutrientes. Es decir, los hogares cada vez con mayor frecuencia acceden a alimentos de alta densidad energética y de menor costo, trayendo como consecuencia ganancias importantes en el peso corporal pero con deficiencia de micronutrientes (Shamah-Levy et al., 2014: 584). A eso debe sumarse la propensión del mercado de alimentos tradicionales en México a la oferta de productos de carga calórica excesiva y, en general, a malas prácticas en la producción de esos productos (como uso de grasas recicladas, optimización de costos por medio de la reducción de la calidad de los insumos). No debe obviarse tampoco causas como "el descenso en la actividad física originado por los modelos de urbanización y el sedentarismo" e, incluso, factores genéticos (Shamah-Levy et al., 2014). Sobra señalar que la tendencia de aumento de la obesidad y el sobrepeso en la población infantil debería 
considerarse, por su importancia, un problema de mayor magnitud que el de la desnutrición, sobre todo por las consecuencias de mediano y largo plazo (Masud Yunes, et al., 2009: 64-65).

México ocupa el cuarto lugar de prevalencia mundial de obesidad infantil, con aproximadamente $28.1 \%$ en niños y $29 \%$ en niñas (Saldívar-Cerón et al., 2015). Según la Encuesta Nacional de Salud y Nutrición 2012 (Gutiérrez et al., 2012b), México también presenta una prevalencia de sobrepeso y obesidad en escolares de 5 a 11 años de $19.8 \%$ y de $14.6 \%$, respectivamente $(34.4 \%$ en total). (Saldívar-Cerón et al., 2015: 56). Un niño con sobrepeso posiblemente será un adulto con sobrepeso y pueden incrementarse sus probabilidades de padecer diabetes mellitus -o alguna otra patología asociada al sobrepeso.

En el caso de los jóvenes, casi la totalidad de las muertes en ambos sexos son causadas, en primer lugar, por homicidios. En segundo lugar, por accidentes de vehículo, y en tercer lugar, por suicidios (CONAPO, 2012). En México, la mortalidad a causa del suicidio se ha incrementado en los últimos cuarenta años (Borges et al., 2012). De acuerdo con datos de la Secretaría de Salud, el suicidio aumentó $215 \%$ entre 1970 y 1998 (Mondragón et al., 2001). De éstos, el $40.8 \%$ de los suicidios ocurre en jóvenes de 15 a 29 años, entre ellos, la tasa alcanza 7.5 suicidios por cada 100 mil jóvenes. Además, es importante tener en cuenta que, del total de suicidios ocurridos en 2013, $81.7 \%$ fueron consumados por hombres y $18.2 \%$ por mujeres (Instituto Nacional de Estadística Geografía, 2015).

En el caso de los accidentes automovilísticos, el alcohol se encuentra en el primer lugar de causa de accidentes, mientras que el segundo lugar lo ocupa el exceso en los límites de velocidad. De acuerdo con información del -en el ámbito nacional-, durante 2012, $11.6 \%$ de los jóvenes reportaron haber tenido un accidente de tránsito bajo los efectos del alcohol (Instituto Nacional de Estadística Geografía, 2014a). Al igual que en el caso del suicidio, la incidencia del consumo de alcohol -que en este punto se vincula con las muertes por accidente de tránsitose presenta en mayor medida en los individuos del sexo masculino. Pese a que debe considerarse que el consumo de alcohol no implica la manifestación de alcoholismo, el inicio de su consumo a edades tempranas puede desarrollar proclividad al consumo de largo plazo y excesivo, pues, por un lado, la permisividad del entorno familiar sienta las bases para considerar el consumo de alcohol como una actividad aceptable socialmente y, por otro, el desarrollo humano durante la adolescencia -con las consecuencias sociales que éste conlleva- refuerza la idea de la aceptabilidad social del consumo de bebidas em- briagantes. Es decir, el consumo en ese segmento de jóvenes probablemente sea un indicador de alcoholismo en el mediano o largo plazo (González \& Alcalá: 2-3; Instituto Nacional de Estadística y Geografía, 2011; Pons y Berjano, 1999: 63-96).

Otro problema que se identifica en el grupo poblacional de los jóvenes es el sobrepeso. De acuerdo con los resultados de la ENSANUT 2012, $35 \%$ de los jóvenes tienen sobrepeso u obesidad. Además, la encuesta indica que más de uno de cada cinco adolescentes tiene sobrepeso y uno de cada diez presenta obesidad (Gutiérrez et al., 2012b: 169). La obesidad, como se verá más adelante también está relacionada con el consumo del alcohol y, posteriormente, como originadora de diabetes mellitus. Es preciso destacar que la manifestación de obesidad en edades tempranas es un factor de riesgo importante para el desarrollo de ciertas patologías, ya en la edad adulta. Así, la obesidad es un factor de riesgo de enfermedades cardiovasculares, cerebrovasculares, hipertensión arterial y diabetes (Campos Nonato et al., 2012: 328-337).

En el grupo de población identificado como adultos, que corresponde a la población entre 30 y 64 años se identifican tres grupos de enfermedades que concentran la mitad de los años de vida asociados con discapacidad (AVD) en los hombres. Los trastornos mentales (25\%), los problemas musculoesqueléticos (19\%) y los problemas neurológicos (7\%) (Lozano, et al., 2013: 583). Por otro lado, las cuatro principales causas de muerte en adultos hombres entre 30 y 59 años de edad son: diabetes mellitus, cirrosis y otras enfermedades crónicas del hígado, homicidios y enfermedades isquémicas del corazón (CONAPO, 2012). En el caso de las mujeres adultas, la mitad de los años de vida asociados con discapacidad (AVD) se concentran en las enfermedades mentales (23\%) y musculoesqueléticas $(23 \%)$, mientras que si se analizan las causas de muerte de entre 30 y 59 años, los primeros cuatro lugares los ocupan la diabetes mellitus, las enfermedades isquémicas del corazón, el cáncer de mama y la cirrosis y otras enfermedades crónicas del hígado. No es menor tener en cuenta que estas enfermedades -tanto las que inciden en hombres cuanto las que lo hacen en la población femenina- se encuentran entre las principales causas de muerte en México (Organización Panamericana de la Salud, 2012: 517).

Como se puede ver, tanto hombres como mujeres comparten la diabetes mellitus, las enfermedades isquémicas del corazón, la cirrosis y otras enfermedades del hígado como causales de muerte, aunque en diferentes grados que se expondrán a continuación. Por otro lado, cuando se analizan las enfermedades actuales -es decir, verificadas actualmente- en am- 
bos sexos, los trastornos mentales ocupan el primer lugar y los musculoesqueléticos el segundo.

En el caso de los adultos, del total de la población de adultos en México, $9.17 \%$ reportó tener un diagnóstico previo de diabetes por un médico, lo que equivale a 6.4 millones de personas. La diabetes en México es la principal causa de muerte. De acuerdo con los datos del informe Salud en las Américas, la diabetes sacarina (diabetes mellitus) se ha mantenido desde 2005 como la causa de muerte número uno, con un aumento continuado en la incidencia de casos (Organización Panamericana de la Salud, 2012: 517).

En el caso de la cirrosis que, como se mencionó, es un problema entre la población adulta, esta está relacionada directamente con el consumo de alcohol. Entre 2000 y 2012, se observó un aumento en el porcentaje de adultos que consumen alcohol (de $39.7 \%$ en 2000 a 34.1 en 2006 y $53.9 \%$ en 2012 , que implica una diferencia estadísticamente significativa). Entre los hombres, el consumo pasó de $56.1 \%$, en el año 2000, a $67.8 \%$, en 2012. El cambio también fue claro entre las mujeres, puesto que $24.3 \%$ de ellas consumía alcohol en 2000 y para 2012 este indicador se elevó a 41.3\% (Guerrero-López et al., 2013: 284). Lo anterior resulta de suma importancia porque la mortalidad atribuible a enfermedades hepáticas, relacionadas con el consumo de alcohol, presenta una tendencia constante entre 2000 y 2010 y se sitúa en 18,192 en promedio por año. Es importante tener en cuenta, sin embargo, que la mortalidad en hombres, por esas causas, es casi cinco veces mayor que la mortalidad en mujeres: 14,868 muertes anuales en promedio para los hombres y 3,322 para las mujeres. (Guerrero-López et al., 2013: 285).

Analicemos ahora el cáncer en México. Las tasas de mortalidad anual por cáncer para todas las edades, en 2010 y 2011, mantuvieron un comportamiento similar: 77.7 y 71.8 por cada 100 mil en hombres, y; 78.5 y 72.8 por 100 mil, en mujeres, respectivamente. Sin embargo, el riesgo de morir por cáncer entre las mujeres fue $44 \%$ mayor en comparacion con los hombres, principalmente en el grupo de 35 a 64 años (Torres-Sánchez et al., 2014: 477). Es interesante resaltar que, al igual que el resto de las enfermedades crónico degenerativas analizadas, el cáncer también está asociado al consumo de alcohol. El consumo de alcohol es un factor de riesgo para diferentes tipos de cáncer, entre ellos, el tumor maligno de hígado y el cáncer de colon y recto. Además, es importante tener en cuenta el papel que pueden jugar los cambios dietéticos en la probabilidad de incidencia de cáncer. México se encuentra entre los países con más altos patrones de consumo de alcohol en el mundo y, durante los últimos 50 años, ha mostrado un incremento sostenido en su consumo. El principal determinante del cáncer de hígado es la cirrosis hepática, asociada con el consumo de alcohol. A éste sigue, en segundo lugar, la infección por el virus de la hepatitis, que también es una patología común en el país (Torres-Sánchez et al., 2014: 487).

El cáncer de mama es la tercera causa de muerte entre las mujeres en México. Algunos factores de riesgo para el cáncer de mama son las dietas hiperenergéticas que resultan en un consecuente sobrepeso u obesidad. Estudios recientes recolectaron datos relacionados con el estado nutricional de pacientes con cáncer de mama y encontraron que casi la totalidad de la muestra evaluada (86\%) presentaba sobrepeso u obesidad. De la misma manera, se encontró que las pacientes evaluadas (97\%) en este proyecto se caracterizaron por presentar una distribución de grasa androide que se refleja en una circunferencia abdominal mayor a la recomendada para la población mexicana. Estas condiciones, propias del Síndrome Metabólico (o Síndrome $\mathrm{X}$ ), tienen incidencia en el desarrollo de otras patologías, como la diabetes o la hipertensión. En México, una de cada dos personas padecen el Síndrome Metabólico y la prevalencia es más elevada en mujeres que en hombres (Cordero-Hernández y PintoAlmazán, 2014: 17). Esto es importante, ya que las anormalidades metabólicas y endocrinas características de la obesidad son más pronunciadas en personas con una distribución androide, es decir, aquellas en las cuales la grasa tiende a acumularse en el abdomen. De hecho, se ha establecido que la distribución androide agrava el riesgo a desarrollar cáncer de mama, ya presente por la obesidad, y que existe una asociación positiva entre la adiposidad abdominal -definida tanto por ICC como por circunferencia abdominal-y el riesgo de desarrollar carcinoma mamario (Gutiérrez et al., 2012a: 189).

En la población de adultos mayores los tres padecimientos con el mayor autorreporte de diagnóstico médico, en las personas del sexo masculino, fueron: hipertensión $(40.0 \%)$, diabetes $(24.3 \%)$ e hipercolesterolemia (20.4\%). Los principales padecimientos entre población femenina son: hipertensión $(40 \%)$, diabetes $(24 \%)$ e hipercolesterolemia $(20 \%)$. Para los indicadores de salud mental: $17.6 \%$ presentó síntomas depresivos; $7.3 \%$, deterioro cognitivo y $7.9 \%$, demencia. Para el estado funcional: $26.9 \%$ reportó dificultad para realizar actividades básicas y $24.6 \%$, para actividades instrumentales (Manrique-Espinoza et al., 2013: 323).

Tanto para hombres como para mujeres de la tercera edad las enfermerdades crónico-degenerativas más padecidas son; en primer lugar la hipertensión, en segundo lugar la diabetes, en tercer lugar la hipercolesterolemia y en cuarto lugar la depresión 
(Manrique-Espinoza et al., 2013).

$\mathrm{Al}$ analizar las causales de muerte en adultos mayores se observa lo mismo, enfermedades crónico degenerativas en los primeros lugares. Para el caso de hombres adultos mayores de 60 años, se encuentran en primer lugar las enfermedades isquémicas del corazón; en segundo lugar, diabetes mellitus; en tercer lugar, enfermedades cerebrovasculares, y; en último lugar, las enfermedades pulmonares obstructivas. Para el caso de mujeres las causales son las mismas, pero en diferente orden. El primer lugar lo ocupa la diabetes mellitus; el segundo, las enfermedades isquémicas del corazón; el tercer lugar las enfermedades cerebrovasculares, y; el último lugar, las enfermedades pulmonares.

En síntesis los principales problemas epidemiológicos que afectan la salud de la niñez en México son la desnutrición, la anemia, la mortalidad infantil y la obesidad. En el caso de los jóvenes, los principales problemas de salud están relacionados con la depresión, el consumo del alcohol y drogas y se reflejan sobre todo en un elevado número de muertes por homicidios, accidentes de vehículo y suicidios. En el caso de los adultos y los adultos mayores los principales problemas son de enfermedades crónico degenerativas tales como la diabetes mellitus, la cirrosis, las enfermedades isquémicas del corazón, las enfermedades cerebrovasculares y el cáncer. Prácticamente todas estas enfermedades están relacionadas con el consumo de alcohol y la obesidad. Por consiguiente, la prevalencia de padecimientos crónico-degenerativos es -y seguirá siendo en el mediano plazo, al menos- la base de las causas de mortalidad en el país. Así, la estructura del gasto público en materia de salud no puede limitarse a la atención paliativa de los padecimientos de ese tipo, pues la presencia de obesidad y sobrepeso en los segmentos jóvenes de la población, así como el consumo de alcohol y tabaco, por ejemplo, delinea un panorama sombrío para los servicios de salud en el país.

Al perfil epidemiológico general que hemos esbozado debe añadirse las proyecciones demográficas. Un sistema de salud debe prepararse no solamente para los problemas que enfrenta en el presente, sino también para los que enfrentará en el futuro. Por lo tanto, en materia de salud y decisiones públicas, es importante considerar la estructura poblacional de mediano y largo plazo de la sociedad que se esté atendiendo.

En México existe un proceso acelerado de envejecimiento que está transformado la estructura poblacional, incrementando la esperanza de vida y modificando el perfil epidemiológico de la población mexicana, de acuerdo con las estimaciones poblacio- nales. Los descensos en la fecundidad y la mortalidad han incidido sobre la magnitud y la naturaleza de las causas de muerte, han sustituido los grupos más afectados y recorrido el momento de la muerte a edades cada vez más avanzadas. De acuerdo con el último censo, en 2010 la población total de México era de 113.5 millones de habitantes (50.7\% mujeres y $49.3 \%$ hombres), derivado de una tasa de crecimiento de $1.5 \%$ anual entre 1990 y 2010 . Por su parte, en el segmento de población de adultos mayores se registraron 10 millones de individuos de 60 años o más, lo que representa el $9 \%$ del total de la población. Su tasa de crecimiento anual es superior a la de la población total, $3.8 \%$, lo que implica que en 2020 habrá casi 16 millones de adultos mayores en el país. En la siguiente gráfica se observa con mayor claridad la inversión de la pirámide poblacional. Como se puede ver, durante la década de los setenta y el 2000, la pirámide presenta una forma triangular en la que la base, compuesta por jóvenes, es la que predomina. En 2030 la pirámide adopta una forma rectangular en la que los adultos mayores comienzan a ser la población más abudante. Finalmente, las proyecciones muestran que para 2050 , los adultos mayores serán el grupo poblacional más grande por mucho.

En la actualidad hay más individuos mayores de 60 años que niños menores de 4 años y las proyecciones indican que el fenómeno del envejecimiento demográfico es irreversible en el mediano plazo, puesto que persistirá durante varias generaciones. Este crecimiento también es una muestra del aumento en la esperanza de vida, que actualmente es de 76.4 años para la población en general. Sin embargo, la esperanza de vida saludable para la población en general es de 65.8 años, lo que significa que los individuos viven 10.6 años con una carga de enfermedad y dependencia (Manrique-Espinoza et al., 2013: 323). Las causas de esos años de dependencia se encuentran en el perfil epidemiológico que hemos detallado. Cada vez más, las enfermedades crónico-degenerativas han sustituido a las enfermedades transmisibles como causales de dependencia y muerte.

Si la población mexicana ahora vive más años y, en promedio, muere a edades mayores, ese incremento en la esperanza de vida no necesariamente garantiza un buen estado de salud de las personas, pues el estudio de carga de la enfermedad de 2010 estimó que México presentaba una esperanza de vida saludable (sin discapacidad) de 67.1 años en las mujeres y de 63.6 años en los hombres, lo que significa que las mujeres viven 11.3 años de su vida -casi siempre la última etapa-con discapacidad y los hombres aproximadamente 10 años (Lozano et al., 2013: 583). Lo anterior implica una carga sustancial para el sistema de salud porque, en unos 
Figura 2: Pirámides demográficas México a través de los años

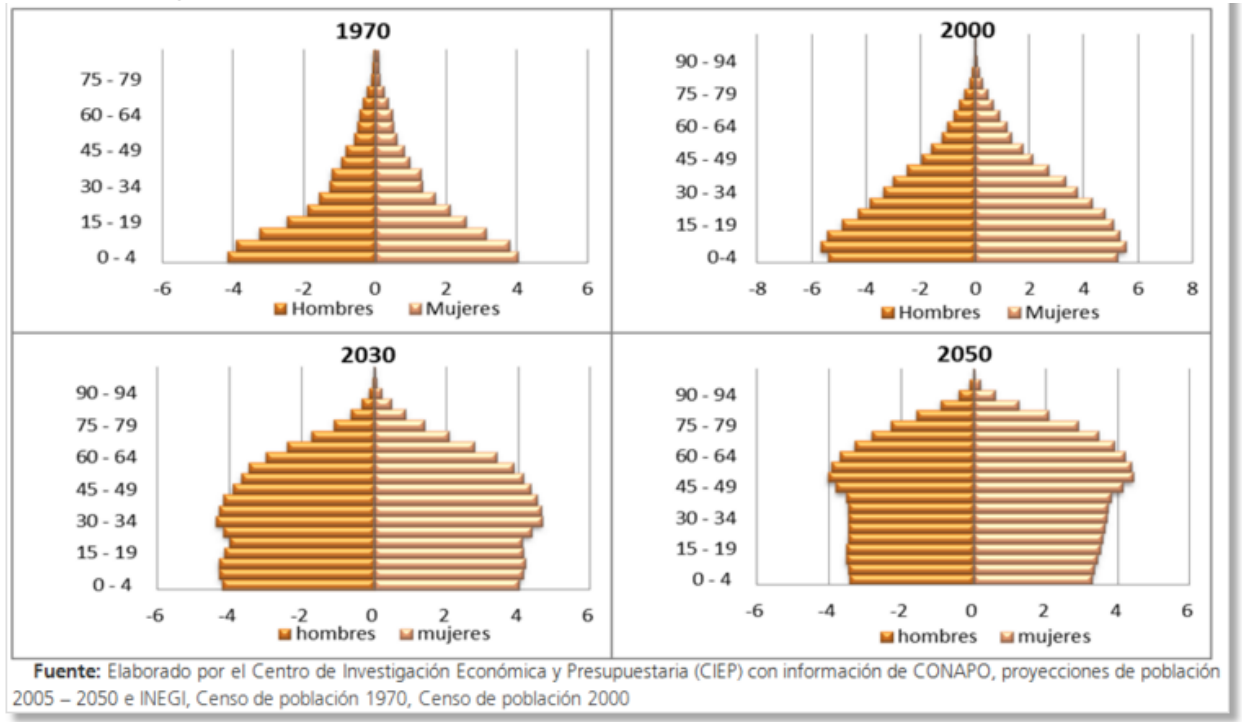

Fuente: Maya 2012

años, la mayor parte de la población será de adultos mayores viviendo más años con alguna enfermedad crónico-degenerativa. En esa previsión se identifican dos problemas para el sistema de salud: (1) la obligación de hacerse cargo de adultos mayores con padecimientos crónico-degenerativos, y; (2) la persistencia de esa obligación durante varios años, al menos una década. La creciente prevalencia de las enfermedades crónicas constituye un reto particular, pues compromete la funcionalidad, genera discapacidad, eventualmente conduce a la dependencia y representa, además, un incremento significativo en el costo de la atención a la salud. Se debe reconocer, por tanto, la importancia del estado funcional de los adultos mayores, ya que determina en gran medida la relación entre el envejecimiento poblacional y el gasto en salud (Manrique-Espinoza et al., 2013: 324).

Como se mencionó al inicio, cuando la política pública se conceptualiza como respuesta o solución a problemas sociales, ésta se convierte en una hipótesis causal, una acción -de características instrumentales- cuyos efectos empíricos producirían una situación social con atributos deseables (Aguilar, 2010: 32). En este sentido, identificados los distintos problemas epidemiológicos y sus causales, se esperaría que el diseño presupuestal estuviera dirigido a su solución. $\mathrm{O}$ al menos a dos ámbitos de acción que, en el futuro, podrían prefigurar una solución: la atención a la población ya diagnosticada y la prevención para tratar de detener el incremento de enfermos crónico-degenerativos. El énfasis en la prevención, además de ser deseable para la política pública de salud, dado el perfil epidemiológico del país, está consagrado en la Ley General de Salud desde el artículo 2, dedicado a detallar el derecho a la protección social en salud. Asimismo, el artículo 27 de ese ordenamiento caracteriza los servicios básicos para la materialización del derecho a la protección social en salud y, entre ellos, se encuentra la prevención de enfermedades de atención prioritaria, transmisibles y accidentes. En todo caso, el análisis también remite a la necesidad de definir con claridad a todos los padecimientos del perfil epidemiológico actual del país como de atención prioritaria.

\section{¿Inercialidad de los ramos des- tinados a la salud o programas presupuestarios como hipótesis causal? Análisis del presupues- to de salud pública en México}

En México, los Ramos destinados a la atención de la salud pública se encuentran dentro de los primeros lugares respecto a presupuesto en los últimos cinco años. De los 43 Ramos analizados, el IMSS se encuentra en cuarto lugar y el ISSSTE en séptimo lugar. Aunado a ello, en los últimos cinco años no se observan grandes variaciones entre los programas presupuestarios de estos 3 Ramos. En el caso del Ramo 12, correspondiente a Secretaría de Salud ha habido sólo dos variaciones en programas en cinco años. En el IMSS ninguna y en el ISSSTE una. Al analizar el número de programas nuevos por Ramo por Año observamos que el Ramo 12, correspondiente a la Secretaría de Salud, sólo registra 9 pro- 
gramas nuevos en los últimos cinco años, el ramo 50 correspondiente al IMSS sólo 1 y el Ramo 51 correspondiente al ISSSTE también sólo uno. Estos datos nos dan mucha luz sobre la inercialidad del presupuesto en el sector salud. Como mencionamos, esta inercialidad podría no ser un problema si el gasto estuviera dirigido a la atención de la prevención de enfermedades crónico degenerativas por ejemplo a la activación física, a la reducción del consumo de alcohol, o a la atención de temas de nutrición y a la disminución de la obesidad desde la niñez y en todos los grupos etarios.

En México, la inversión pública en salud desde 2010 ha pasado de $2.4 \%$ a $3.2 \%$ del PIB en 2016 (OCDE, 2016) y en los próximos años continuará incrementándose como consecuencia del perfil epidemiológico y poblacional que tendrá el país. En lo que respecta a los ramos del PEF que atienden el tema de salud se utilizan los siguientes para el análisis de hipótesis causal ${ }^{2}$ : Ramo 12, Secretaría de la Salud; Ramo 50, IMSS; y Ramo 51, ISSSTE.

En términos de presupuesto aprobado, el Ramo 12 del PEF, que corresponde a la Secretaría de Salud, pasó de 89.9 mil millones de pesos en 2010 a 134.9 mil millones de pesos en 2015, lo que representa un incremento en términos reales del $20 \%$. En el caso del Ramo 50 del PEF, que corresponde al IMSS, el presupuesto en este mismo periodo pasó de 320.3 mil millones de pesos en 2010 a 497.6 mil millones de pesos en 2015, un incremento real del 24.28 \%. En el caso del ISSSTE, Ramo 51 del PEF, el presupuesto pasó de 107 mil millones de pesos en 2010 a 208 mil millones de pesos en 2015, un incremento real del $55 \% .^{3}$.

Las preguntas guía de esta sección, son las siguientes: ¿cuál es la relación entre estos programas presupuestales y los principales padecimientos y sus causas en los distintos estratos de edad de la población?,¿con qué criterios y de qué forma se utiliza el gasto destinado a la prevención de los principales padecimientos?

A continuación se analizarán los problemas epidemiológicos en los niños y las acciones presupuestales de los programas que los atienden. Como se identificó en la segunda sección, en ese grupo de población (niños) uno de los principales problemas es la inseguridad alimentaria que se asocia con la desnutrición, la anemia, la talla y la obesidad. Estos

\footnotetext{
${ }^{2}$ Es importante tener en cuenta que existen recursos asignados fuera de estos tres ramos que podrían también indirectamente abordar la problemática, sin ser su prioridad. Sin embargo, como se mencionó, se analizaron estos tres ramos porque son los únicos que tienen como principal prioridad velar por la salud pública nacional.

${ }^{3}$ Los incrementos se calcularon con base en una inflación de enero 2010 a enero 2015 del $20.07 \%$ según INEGI
}

problemas se acentúan en niveles socioeconómicos bajos y en población indígena. En el Ramo 12, correspondiente a la Secretaría de Salud, se identificó sólo un programa que, entre sus muchos otros objetivos, asigna un espacio al problema de la desnutrición en los sectores menos favorecidos, el programa Prospera ${ }^{4}$. Este programa consiste en acciones de salud dirigidas a la comunidad y suplementos alimenticios. A Prospera le corresponde $4.47 \%$ del total del presupuesto del Ramo 12 (2015). Otra parte de la asignación presupuestal, que también beneficia a la niñez, aunque no se dirige a los causales de los principales problemas epidemiológicos que enfrentan actualmente, es el de vacunación. Para este propósito, en el Ramo 12 hay un programa cuya asignación representa el $1.42 \%$ del presupuesto total del Ramo en 2015.

Además de Prospera y el programa de vacunación, se identificaron tres programas dirigidos específicamente a los niños, sin embargo, los programas tienen que ver más con el derecho al acceso a la salud y derechos humanos que con las principales causales de los problemas epidemiológicos que se presentan en este segmento de edad. Por ejemplo, se identificó el Programa para la Protección y el Desarrollo Integral de la Infancia ${ }^{5}$, el Programa de Fortalecimiento a las Procuradurías de Defensa del Menor y la Familia, así como el Seguro Médico Siglo XXI, que es una especie de Seguro Popular para niños con fines sobre todo curativos.

En el Ramo 50 que corresponde al IMSS, hay un solo programa dirigido a este grupo etario, el de "Servicios de Guardería", que representa el 1.94\% de los recursos aprobados. Sin embargo, al igual que lo que sucede en el Ramo 12, el programa no se dirige a la solución de los problemas epidemiológicos específicos que enfrenta esta población en esta edad. En el caso del Ramo 51, que corresponde al ISSSTE, se identifican tres progamas dirigidos a la niñez pero nuevamente, los programas no tienen como objetivo solucionar los problemas epidemiológicos que padecen. El "Programa de control de enfermedades prevenibles por vacunación", el "Programa de atención materno infantil" y el de "Servicios de Estancias de Bienestar y Desarrollo Infantil". En este Ramo además, se identificó un programa que se dirige al tema de la inseguridad alimentaria, el "Programa y servicio de apoyo para la adquisición de productos básicos y de consumo para el hogar". Cabe aclarar, que el programa es para las familias en general y no para la niñez en específico. Estos

\footnotetext{
${ }^{4}$ Hay que señalar que este programa también es operado y ejerce recursos en otros ramos del presupuesto federal, como el de desarrollo social o educación.

${ }^{5}$ Este programa está a cargo del Sistema Nacional para el Desarrollo Integral de la Familia. Los recursos del programa se ejecutan por medio de los sistemas estatales.
} 
cuatro programas representan el $1.58 \%$ del presupuesto del Ramo.

$\mathrm{Al}$ igual que lo que sucede con el resto de los grupos poblacionales, en esta edad no se observa en la Secretaría de Salud, en el IMSS y en el ISSSTE, un énfasis en la prevención vía programas nutricionales para solucionar los problemas de inseguridad alimentaria, desnutrición y obesidad que se observan sobre todo en los niveles socioeconómicos bajos. El Seguro Popular, por ejemplo, que forma parte del Ramo 12, entre sus cientos de espacios de cobertura incluye uno para acciones preventivas en la niñez por grupos quinquenales, sin embargo, se desconocen las acciones específicas en este sentido. Las cifras mencionadas evidencian la incapacidad que ha habido hasta el momento para diseñar una estructura de gasto que atienda las principales causas de los problemas epidemiológicos del grupo poblacional correspondiente a niños. Cuando se identifica un problema público transversal y las acciones de política pública se dirigen a atender de forma muy general los distintos ámbitos de ese problema, frecuentemente sucede lo que se puede observar en el Ramo 12, 50 y 51 del PEF: la capacidad del gasto federal para atender las causales de los problemas epidemiológicos de la infancia se diluye entre varias líneas de acción que no necesariamente atienden esas causas.

La población indígena $1^{6}$, por su parte, que como mencionamos, padece en mayor medida problemas de inseguridad alimentaria, desnutrición y talla no se ve favorecida por una mayor cantidad de programas o presupuesto en materia de salud. En el Ramo 12, no se identificó ningún programa para atender a esta población. Existen, por ejemplo, programas como el de Caravanas de la Salud que benefician a la población indígena, ya que se dirigen a los sectores poblacionales con "más hambre", sin embargo, el énfasis no está en la niñez indígena ${ }^{7}$. En los programas que opera la Comisión Nacional para el Desarrollo de los Pueblos Indígenas (CDI), que está sectorizada en la Secretaría de Hacienda y Crédito Público, existen líneas de acción que podrían atender las desventajas de la atención integral a la niñez indígena, pero su énfasis es general y no específico. En el resto de los ramos analizados (50 y 51) no existen programas dirigidos específicamente a es-

\footnotetext{
${ }^{6}$ Según datos del Censo de Población y Vivienda 2010, había en México 6.6 millones de personas indígenas, consideradas así por la característica de ser hablantes de una lengua originaria. De esos, el $61 \%$ residía en poblaciones de menos de 2500 habitantes.

${ }^{7}$ Para completar este análisis sin embargo, tendría que analizarse el Ramo 6 del Presupuesto de Egresos de la Federación, ya que en la Secretaría de Hacienda y Crédito Público existen decenas de programas dirigidos a población indígena. Habría que analizar, cuántos y cuáles de ellos están relacionados con el tema de la salud.
}

te grupo poblacional en relación con la nutrición, aunque los programas de Liconsa, Diconsa y PAL de SEDESOL probablemente los beneficien debido a que se dirigen a la población con menos recursos, que en México coincidentemente es también la población indígena ${ }^{8}$.

En el caso de los jóvenes, como hemos señalado, las principales causas de muerte son el homicidio, accidentes automovilísticos y suicidios, todas relacionadas con el consumo de alcohol y otras drogas. En el caso de los suicidios, muchos están relacionados con condiciones como la depresión. El análisis de los programas presupuestarios del Ramo 12, 50 y 51 deja ver la existencia de un descuido a este sector de la población, pues no hay un solo programa dirigido específicamente a los jóvenes. En 2012, en el Ramo 12 había dos programas dirigidos al tema de las adicciones; no obstante, en la actualidad solamente hay uno dirigido a atender este problema de manera general, no exclusivametne dirigido a los jóvenes. El gasto en este programa representó el $1.04 \%$ del total del Ramo 12 , en $2015^{9}$.

Además de estos temas, en los jóvenes también se identificaron problemas de obesidad importantes, sin embargo, como se verá más adelante, en los ramos analizados, la prevención no pareciera ser prioridad. El Seguro Popular, al igual que lo que sucede con el grupo de niños, tiene dentro de su esquema de cobertura "Acciones Preventivas para Jóvenes", sin embargo, se desconoce cuáles son estas acciones, el impacto que tienen y qué porcentaje representan del presupuesto destinado a Seguro Popular ${ }^{10}$. En

\footnotetext{
${ }^{8}$ Es importante tener en cuenta que en otros ramos, como el 20, que corresponde a la Secretaría de Desarrollo Social, se identificaron "5" programas relacionados con aspectos de nutrición, algunos de ellos dirigidos especialmente a la niñez, tales como el Programa de adquisición de leche nacional a cargo de LICONSA, el Programa de abasto social de leche a cargo de LINCONSA y el Programa de abasto rural a cargo de DICONSA. Además, programas no dirigidos específicamente a este grupo etario pero sí al tema de nutrición se identificó el Programa de apoyo alimentario y los Comedores comunitarios. Estos cinco progragamas representan el $11.77 \%$ del presupuesto total de SEDESOL en 2015. Finalmente, otros programas dentro de SEDESOL dirigidos a la niñez en particular aunque no necesariamente relacionados con las causales epidemiológicas analizadas en esta edad se encuentra el Programa de estancias infantiles para apoyar a madres trabajadoras, que se lleva el $3.33 \%$ del presupuesto en 2015. En el Ramo 11, que corresponde a la Secretaría de Educación Pública, existen siete programas relacionados con el deporte, infraestructura para el deporte y cultura física que sí están relacionados con este tipo de problemáticas, su presupuesto corresponde al $1.25 \%$ del total de presupuesto 2015 de la SEP.

${ }^{9}$ No debemos dejar de lado, sin embargo, que entre los cientos de esquemas de cobertura del Seguro Popular existe una Consejería de Adicciones en adolescentes. Sin embargo, su utilidad y funcionamiento se desconocen, además de que, recordemos que el Seguro Popular atiende sólo a un sector de la población joven.

${ }^{10} \mathrm{El}$ acceso al presupuesto desagregado del Seguro Popular
} 
el IMSS y el ISSSTE como se mencionó, no hay un solo programa dirigido a jóvenes en específico y tampoco ninguno para tratar problemas de obesidad. En materia de prevención en el ISSSTE (Ramo 51) existe solamente un programa "Detección oportuna de enfermedades", sin embargo, no está dirigido específicamente a este grupo etario y su presupuesto es del $.09 \%$ del total del presupuesto de este Ramo ${ }^{11}$.

Con respecto a los adultos, el problema de las enfermedades crónico degenerativas afecta principalmente a hombres y mujeres que viven en zonas urbanas, estas enfermedades son: diabetes, problemas cardiacos, cerebrovasculares, cirrosis y cáncer de mama, en el caso de las mujeres. De los 37 programas que componen el Ramo 12 en el 2015, solamente uno tiene como propósito específico atender de manera preventiva estos problemas y significa tan sólo el $0.64 \%$ del presupuesto 2015. En relación con esto, por ejemplo, el gasto en causales como obesidad, tan importantes en estas enfermedades, representa solamente el $0.30 \%$ vía dos programas: Programa Comunidades Saludables y Prevención Contra la Obesidad. En el Ramo 50, que corresponde al IMSS, no se identifica tampoco ningún programa dirigido a la prevención y en el ISSSTE como mencionamos hay solamente un programa dirigido a la prevención, "Detección oportuna de enfermedades", cuyo presupuesto como mencionamos es mínimo. En ambos casos, ninguna mención en materia de obesidad. Los datos anteriores evidencian una falta de atención al tratamiento y no se diga a la prevención de las enfermedades crónicodegenerativas que son una suerte de espada de Damocles para el sistema de salud mexicano. En la información que se reseña en este párrafo, además, es diáfano que la atención preventiva de ese tipo de padecimientos es mínima. Y surge el cuestionamiento acerca de si es más grave -en perspectiva de futuro- la nula prevención de esas enfermedades o el hecho de que las acciones preventivas reciban atención exigua.

El consumo del alcohol es causa de muchas enfer-

resulta complicado. Este tema incluso ha sido durante varios años objeto de demandas por parte de grupos de la sociedad civil en pos de la Transparencia Presupuestaria.

${ }^{11}$ En otros Ramos, como el Ramo 11, que corresponde a la Secretaría de Educación Pública, se identificaron tres programas dirigidos específicamente a los jóvenes. El primero de ellos directamente relacionado con estos problemas, el Programa de reinserción académica de los jóvenes integrantes de bandas y pandillas. El segundo, dirigido también a un problema importante para las mujeres en esta etapa, el Programa de becas de apoyo a la educación básica de madres jóvenes y jóvenes embarazadas. El tercero, el Subsidio a programas para jóvenes, presente también en el Ramo 20 de SEDESOL que atiende transversalmente varios de los problemas que enfrenta la juventud actualmente mediante apoyos para el emprendimiento, vivienda, salud y educación. medades crónico degenerativas y, dentro del Ramo 12, 50 y 51, la prevención de esta problemática ni de lejos ocupa un lugar prioritario. En el Ramo 12, como mencionamos, sólo existe un programa dirigido al problema de las adicciones y su porcentaje es del $1.04 \%$ del gasto total del ramo. En lugar de incrementar el número de programas para atender esta causal, que abarca gran parte de los problemas epidemiológicos del país, en 2013 se decidió disminuir el presupuesto del Ramo 12 y quitar uno de los dos programas que existían. Lo anterior, es evidencia de que el aspecto preventivo no es una prioridad, por lo menos reflejada en el gasto del ramo que nos ocupa, y; en segundo lugar, no se observa una hipótesis causal entre los principales problemas epidemiológicos, sus orígenes y la asignación presupuestal. En el Ramo 50 del IMSS y el Ramo 51 del ISSSTE tampoco se identificó ningún programa para combatir adicciones o reducir el consumo de alcohol entre la población. Es decir, hasta el momento el diseño presupuestal ha ignorado la urgencia de vincular los problemas epidemiológicos actuales y futuros con comportamientos y causas que son posibles de atajar con acciones preventivas. Incluso por lógica económica sería urgente que la planeación presupuestal en materia de salud pusiera atención en la prevención, pues, de seguir en la trayectoria actual, los problemas epidemiológicos que hemos mencionado alcanzarán niveles casi inmanejables en términos de política pública. Y esta predicción no es resultado de proyecciones sociológicas, sino de investigaciones y datos de la ciencia médica mexicana.

Un estudio reciente del Instituto Nacional de Salud Pública (INSP) en el que se estima la carga atribuible a la exposición a diversos factores de riesgo que son determinantes en la aparición de numerosos problemas de salud encontró que, para el caso de los hombres, destacan el consumo de alcohol, el sobrepeso, la obesidad, los niveles elevados de glucosa en sangre, la presión arterial alta y el consumo de tabaco como los factores de riesgo más importantes y responsables de $35.6 \%$ de los años de vida saludable perdidos en 2010 (Lozano et al., 2013: 584). En el caso de las mujeres, los factores que explican las principales enfermedades crónico degenerativas tienen que ver con el sobrepeso, la obesidad, glucosa elevada, la hipertensión, el sedentarismo, el consumo de alcohol y tabaco que, en su conjunto, se responsabilizan por el $40 \%$ de la carga de los años de vida saludable perdidos (Lozano et al., 2013: 584). En ambos casos, el sobrepeso y la obesidad son factores explicativos importantes en materia de prevención. Sin embargo, en los ramos 12,50 y 51 , que son los ramos destinados a la salud, no se identificó ningún programa cuyo objetivo sea atender este problema. Contrario a lo que se espe- 
raría, el único programa que existía en el Ramo 12 entre 2010 y 2011, denominado "Prevención contra la Obesidad", desapareció en 2013 y actualmente no se asignan recursos del ramo 12,50 y 51 para la prevención en este tema. Lo mismo sucede con el tema de la actividad física.

La gran parte de las enfermedades crónicodegenerativas y causales de muerte en todas las edades son prevenibles, lo cual podría ser la esperanza que queda a futuro en términos de sostenimiento del sistema de salud. Los elevados costos curativos de estas enfermedades y la transición demográfica que nos garantiza un incremento significativo de la población adulta mayor que será afectada por ese tipo de padecimientos, podrían poner en riesgo la viabilidad del sistema de salud y de las finanzas públicas mexicanas en su conjunto, pues sería muy complicado atender - con recursos- a los sujetos de esas enfermedades. Es claro que se requiere reorientar el gasto público en salud para prevenir estas enfermedades con la finalidad de que, en un futuro, no alcancen las magnitudes inmanejables que se prevén. Esta dinámica sería difícil de controlar y alteraría -si se manifiesta- el funcionamiento global de las finanzas públicas del país.

Respecto a la brecha en salud entre las zonas urbanas y las zonas rurales, se identificó en el Ramo 12 dos programas que la atienden: el Programa Caravanas de Salud, cuyo porcentaje del presupuesto total es del $0.58 \%$ del total del Ramo 12 y Prospera que, como ya se mencionó, representa $4.47 \%$. De suerte que el vínculo causal que se sugiere establecer ya existe en este caso. Sin embargo, el análisis del Ramo 50 y 51 no refleja este objetivo vía el gasto, es decir, no se observa aún ningún programa con asignación presupuestal cuyo objetivo sea atender esta problemática.

Otro aspecto que se ha dejado de lado, y que afecta a tres grupos poblacionales: jóvenes, adultos y adultos mayores, es el de la salud mental. En el análisis de los 37 programas presupuestarios del Ramo 12 no se encontró un solo programa dirigido a esta problemática en específico. El Seguro Popular, tiene dentro de su esquema de cobertura acciones preventivas como la detección precoz de los trastornos alimentarios, trastornos depresivos, de ansiedad y adicciones; estas acciones están distribuidas por grupos etáreos. Sin embargo, se desconoce cuáles son estas acciones, el impacto que tienen y qué porcentaje representan del presupuesto destinado a Seguro Popular, pues la glosa a ese nivel de detalle no existe en el presupuesto ${ }^{12}$. En el análisis del Ramo

\footnotetext{
${ }^{12}$ Dada la cantidad de acciones que se incluyen dentro del Seguro Popular y el porcentaje de los recursos que se asignan a este Programa en el Ramo 12, sería necesario, para completar este análisis, tener acceso a la manera en que el
}

50 y 51 por su parte, tampoco se observó ningún programa dirigido a atender trastornos mentales.

Por otra parte, un aspecto positivo que se identificó en el análisis, es el gasto que se dirige a adultos mayores en materia preventiva. A diferencia de lo que sucede en otros grupos poblacionales, en el caso de los adultos mayores, el Ramo 12 sí destina recursos a favor de la promoción del envejecimiento sano y activo, vía Seguro Popular y el Catálogo Universal de Servicios de Salud (CAUSES), a través de la inversión en prevención y promoción de la salud y manteniendo una perspectiva de curso de vida. De acuerdo a una investigación reciente (Manrique-Espinoza et al., 2013) actualmente, dentro del CAUSES se incluyen casi todas las acciones preventivas necesarias para este fin. El Seguro Popular se lleva el $55.4 \%$ de todo el presupuesto del Ramo 12. En el Ramo 50 y 51, desafortundamente no se identificaron programas específicos para atender los problemas epidemiológicos de este grupo poblacional. A pesar de que existen programas para adultos mayores, estos se centran en cuestiones como el "Régimen de pensiones y jubilaciones" en el caso del Ramo 50 (IMSS) y "Servicios funerarios" "Pensiones por vejez", "Pagos de funeral", "Pensiones y jubilaciones" en el caso del Ramo 51 (ISSSTE).

Para concluir, es importante hacer un último apunte que abunda en que el aspecto preventivo y las causas de los principales problemas epidemiológicos en el país no son una prioridad en materia presupuestal vía el Ramo 12, 50 y 51. Para ello, se segmentó el gasto del ramo 12 por tema y se observa que $17 \%$ del presupuesto total de los ramos se gasta en cuestiones relacionadas con capacitación de Recursos Humanos, infraestructura y administración; un $18 \%$ está destinado a mejorar la calidad de los servicios de salud que se ofrecen. Si a ello se le suma el $55.4 \%$ del presupuesto que se asigna a Seguro Popular, encontramos que, en estos tres aspectos, se destina el $90.44 \%$ del total del presupuesto del Ramo 12. El gasto en prevención como se puede observar en la siguiente gráfica, es insignificante.

En el Ramo 50 la situación es similar, ya que como se mencionó, no existe ningún programa presupuestal cuyo objetivo sea la prevención o la contención de los problemas epidemiológicos que aquejan a la población en México. Como se puede observar en la Gráfica 5, la gran parte del presupuesto se destina a cuatro programas: Atención curativa eficien-

Seguro Popular distribuye los recursos que le son asignados entre las distintas acciones que realiza así como conocer las evaluaciones, si es que existen, sobre los resultados de las mismas. Desafortunadamente, este es un vacío importante aún en materia de transparencia presupuestaria. 


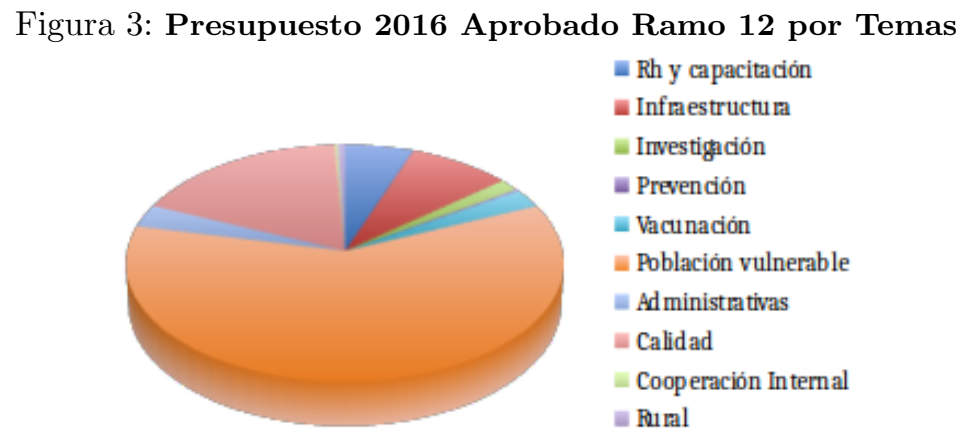

Fuente: elaboración propia con base en Ramo 12 PEF 2015 Aprobado

te, Pensiones en curso de pago Ley 1973, Pensiones y Jubilaciones y Actividades de Apoyo Administrativo. Ninguno de los cuales tiene como objetivo la prevención.

En el Ramo 51, correspondiente al ISSSTE, solamente se identifica un programa dirigido a la prevención "Detección oportuna de enfermedades". Sin embargo, como se mencionó, su presupuesto es del $.09 \%$ del total del presupuesto de este Ramo. Al igual que en el Ramo 50, una parte sustancial del presupuesto del ISSSTE se destina a pago de pensiones y jubilaciones y prácticamente nada del presupuesto a programas dirigidos a la prevención.

El análisis de los tres ramos deja ver la poca importancia que tienen las tareas de prevención de las principales enfermedades crónico degenerativas dados los programas y las asignaciones correspondientes. El análisis de los ramos pareciera indicar que el gasto del Gobierno Federal en materia de salud no tiene como prioridad la atención a los principales problemas epidemiológicos actuales y sus causales. El análisis epidemiológico muestra que el aspecto preventivo debería ser actualmente pritoritario, en cuestiones como el alcoholismo, la nutrición y la obesidad. La evidencia señala que el gasto es muy bajo en estos temas.

\section{Conclusiones}

La intención principal de la investigación fue analizar el sistema de salud en México y su presupuesto en los últimos cinco años. Sobre todo, llamar la atención sobre un problema que atenta contra su estabilidad a futuro: la inercia presupuestal y las proyecciones demográficas de las enfermedades crónico-degenerativas. La lógica de la política pública ofrece elementos para la solución de problemas públicos. En el caso particular de la realidad epidemiológica del país, las previsiones ponen sobre la mesa, con antelación suficiente, los principales detalles de un problema de proliferación de padecimientos crónico-degenerativos. En pocas ocasiones es posible prever la emergencia de un problema público.

El análisis del PEF 2010-2015 en el ámbito de los Ramos destinados a la atención de la salud pública deja dos lecciones importantes. La primera, es que la presupuestación de estos Ramos es inercial y no refleja el rumbo de los problemas epidemiológicos identificados. La segunda, es que la relación causal que se planteó inicialmente no se observa sino por el contrario, deja claro que las prioridades del gasto en materia de salud no están enfocadas a la prevención de las enfermedades crónico degenerativas.

No debe perderse de vista que el gasto en acciones preventivas, empata con la lógica de disminución del gasto futuro y puede considerarse gasto de inversión si se tiene en cuenta que el objetivo de largo plazo es evitar el aumento desproporcionado de la prevalencia de los principales padecimientos crónico-degenerativos que son, a la vez, las principales causas de muerte en el país -y lo serán en el futuro.

La discusión se resume en una idea: la única forma en que la política pública y el diseño presupuestal federal puede incidir positivamente en las previsiones epidemiológicas del país es mediante un cambio de paradigma, un énfasis en la prevención de las enfermedades crónico-degenerativas como gasto de inversión. Desde 1917 Hill escribió respecto a la salud pública y los cambios de paradigmas "la vieja salud pública se ocupaba del ambiente, la nueva se ocupa del individuo. La vieja buscaba la fuente de las enfermedades infecciosas en el entorno del hombre; la nueva las encuentra en el hombre mismo" (Frenk, 2016). En el centro de la solución al incremento de los padecimientos crónico-degenerativos se encuentra la prevención: los hábitos alimenticios, la dieta, la práctica del ejercicio físico y la información. Si bien, los incrementos en el gasto público en salud son importantes, más importante es que esos 
Figura 4: Principales programas y asignación presupuestal 2015 Ramo 50: IMSS

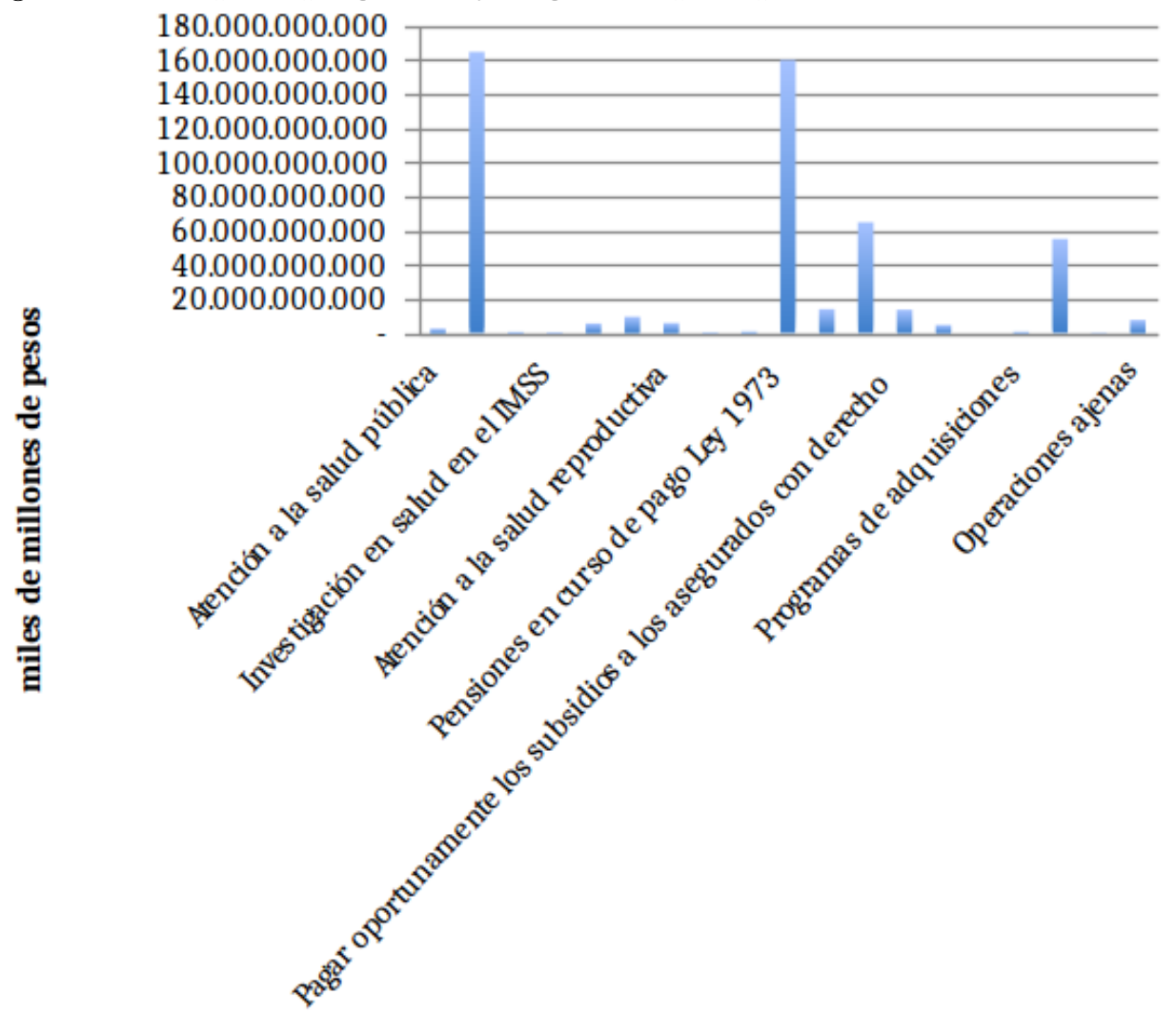

Fuente: Elaboración propia con base en Ramo 50 PEF 2015 Aprobado

Figura 5: Principales programas y asignación presupuestal 2015 Ramo 51: ISSSTE

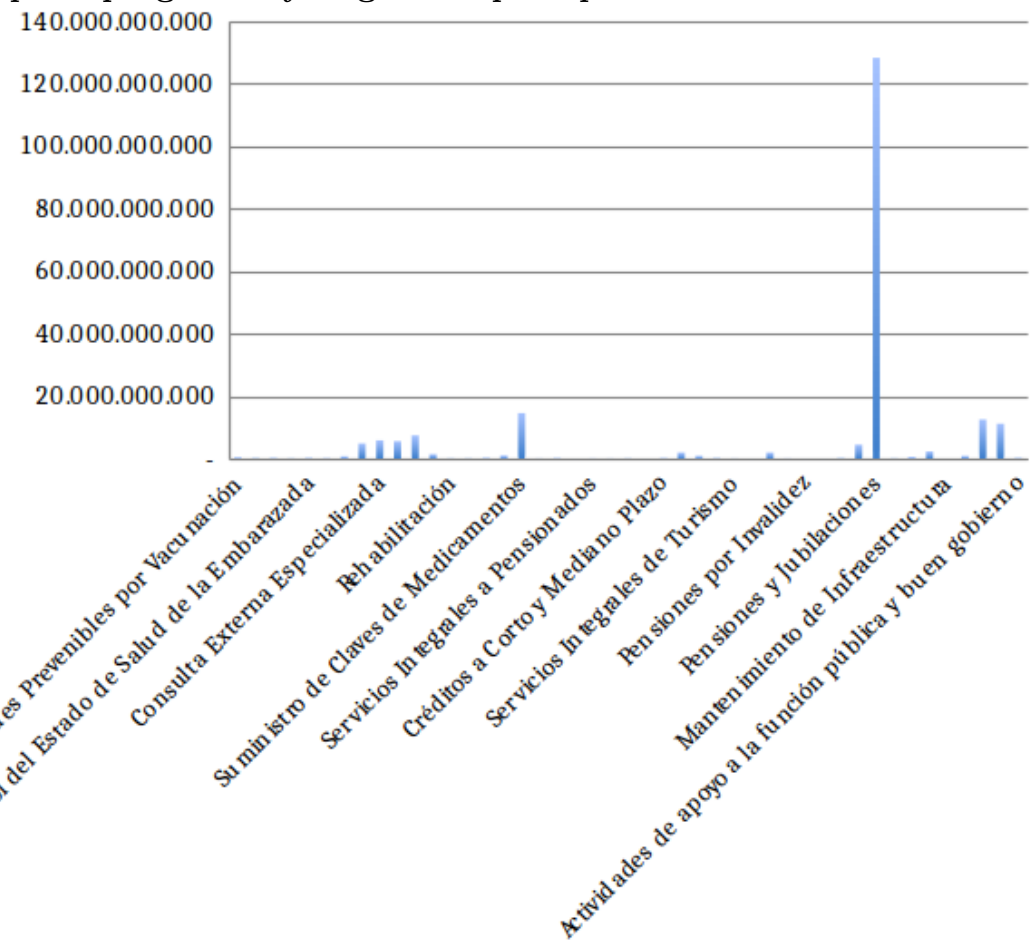

Fuente: Elaboración propia con base en Ramo 51 PEF 2015 Aprobado 
incrementos se reflejen en políticas que no sólo se encaminen a servicios curativos, infraestructura o gasto administrativo, sino que permitan la modificación del énfasis de la política de salud hacia la prevención, único aspecto que contribuye a hacer sostenible el sistema a futuro.

Es posible entender la inercia con que se ha manejado el diseño presupuestal en materia de salud en México, pues las prioridades han pasado varias décadas inalteradas y la dinámica sanitaria del país sigue exigiendo recursos en acciones curativas, sin embargo, las investigaciones médicas -a diferencia de los criterios valorativos de los diseñadores del presupuesto- prefigura un panorama oscuro para la sostenibilidad del sistema sanitario. La gran parte de las enfermedades crónico-degenerativas y causales de muerte son prevenibles. Asimilar el gasto en prevención como gasto de inversión es acaso la mejor forma de comprender que, de la planeación preventiva que se haga ahora, dependerá el perfil epidemiológico de las próximas décadas. Sin embargo, como observamos en esta investigación y como lo han identificado también organismos internacionales, el gasto en administración (burocracia) de las instituciones de salud, en México, es uno de los más altos de la OCDE (OCDE, 2015).

Si cabe, como una línea transversal adicional, es preciso tener en cuenta que la sujeción de los objetivos de la política de salud en México a los planes gubernamentales de cada sexenio hace complicado establecer estrategias de largo o mediano plazo. Eso es claro cuando se observa que la planeación presupuestal ha seguido la inercia de los gobiernos anteriores, sin alterar las estrategias transexenales como el Seguro Popular-, pero tampoco modificando sus objetivos, de modo que esos programas que trascienden los períodos de gobierno aprovechen esa condición para atacar con constancia y consistencia los problemas epidemiológicos del país.

\section{Referencias}

Aguilar, L. (1992). La hechura de las Políticas. Miguel Ángel Porrúa, México D. F.

Aguilar, L. (2010). Introducción. En Política Pública. Miguel Ángel Porrúa, México D. F.

Berry, W. D. (1990). The confusing case of budgetary incrementalism: Too many meanings for a single concept. The Journal of Politics, 52(1):167-196.

Borges, G., Orozco, R., y Medina Mora, M. E. (2012). Índice de riesgo para el intento suicida en México. salud pública de méxico, 54(6):595606 .
Campos Nonato, I., Barquera, S., y Aguilar, C. A. (2012). Efecto de la obesidad sobre otras enfermedades crónicas y la mortalidad. En Rivera Dommarco, J. Á., Hernández-Ávila, M., Aguilar-Salinas, C. A., Vadillo-Ortega, F., y Murayama-Rendón, C., editores, Obesidad en México: recomendaciones para una política de Estado. UNAM, México D. F.

Cordero-Hernández, A. y Pinto-Almazán, R. (2014). Diabetes mellitus tipo 1 y 2. Estudio epidemiológico del primer año del servicio de Consulta Externa del Hospital Regional de Alta Especialidad de Ixtapaluca. diabetes, 3:10.

Cuevas-Nasu, L., Rivera-Dommarco, J. A., Shamah-Levy, T., Mundo-Rosas, V., y MéndezGómez Humarán, I. (2014). Inseguridad alimentaria y estado de nutrición en menores de cinco años de edad en México. Salud pública de méxico, 56:47-53.

Frenk, J. (2016). La salud de la población.: Hacia una nueva salud pública. Fondo de Cultura Económica.

Guerrero-López, C. M., Muños-Hernández, J. A., Sáenz de Miera-Juárez, B., Pérez-Núñez, R., y Reynales-Shigematsu, L. M. (2013). Impacto del consumo nocivo de alcohol en accidentes y enfermedades crónicas en México. Salud pública de méxico, 55:282-288.

Gutiérrez, G., Autrique, M., y Ceballos, G. (2012a). Sobrepeso y obesidad: frecuentes características del estado nutricional en una muestra de pacientes mexicanas con cáncer de mama localmente avanzado. Revista Colombiana de Cancerología, 16(3):187-191.

Gutiérrez, J. P., Rivera-Dommarco, J., ShamahLevy, T., Villalpando-Hernández, S., Franco, A., Cuevas-Nasu, L., Romero-Martínez, M., y Hernández-Ávila, M. (2012b). Encuesta Nacional de Salud y Nutrición. Resultados Nacionales. Instituto Nacional de Salud Pública (MX), Cuernavaca.

Instituto Nacional de Estadística Geografía (2014a). Perfil sociodemográfico de jóvenes. INEGI, México D. F.

Instituto Nacional de Estadística Geografía (2014b). Perfil sociodemográfico de niños. INEGI, México D. F.

Instituto Nacional de Estadística Geografía (2015). Estadísticas sobre suicidio en México. INEGI, México D. F.

Instituto Nacional de Estadística y Geografía (2011). Encuesta Nacional de Adicciones 2011. Alcohol. INEGI, México D. F. 
Lindblom, C. E. (1979). Politics and markets: The world's political-economic systems. Business Horizons, 22(5):80-81.

Lozano, R., Gómez-Dantés, H., Garrido-Latorre, F., Jiménez-Corona, A., Campuzano-Rincón, J. C., Franco-Marina, F., Medina-Mora, M. E., Borges, G., Naghavi, M., y Wang, H. (2013). La carga de enfermedad, lesiones, factores de riesgo y desafíos para el sistema de salud en México. salud pública de méxico, 55(6):580-594.

Manrique-Espinoza, B., Salinas-Rodríguez, A., Moreno-Tamayo, K. M., Acosta-Castillo, I., SosaOrtiz, A. L., Gutiérrez-Robledo, L. M., y TéllezRojo, M. M. (2013). Condiciones de salud y estado funcional de los adultos mayores en México. salud pública de méxico, 55:S323-S331.

Mondragón, L., Borges, G., y Gutiérrez, R. (2001). La medición de la conducta suicida en México: estimaciones y procedimientos. Salud Mental, $24(6)$.

OCDE (2015). Estadísticas de la OCDE sobre la salud 2014. México en comparación.

OCDE (2016). Estudios de la OCDE sobre los sistemas de salud: México 2016.

Organización Panamericana de la Salud (2012). Health in the Americas: 2012 Edition. Regional Outlook and Country Profiles. PAHO, Washington D.C.

Pons, J. y Berjano, E. (1999). El consumo abusivo de alcohol en la adolescencia: Un modelo explicativo desde la psicología social. Plan Nacional sobre Drogas, Valencia.

Saldívar-Cerón, H. I., Ramírez, A. G., Acevedo, M. A. R., y Pérez-Rodríguez, P. (2015). Obesidad infantil: factor de riesgo para desarrollar pie plano. Boletín médico del Hospital Infantil de México, $72(1): 55-60$.

Shamah-Levy, T., Mundo-Rosas, V., y RiveraDommarco, J. A. (2014). La magnitud de la inseguridad alimentaria en México: su relación con el estado de nutrición y con factores socioeconómicos. Salud pública de méxico, 56:79-85.

Székely, M. (2013). Evaluación Externa de los Resultados del Consejo Nacional de Evaluación de la Política de Desarrollo Social. Instituto de Innovación Educativa del Instituto Tecnológico y de Estudios Superiores de Monterrey, México D. F.

Tapia Téllez, G. (2016). Incrementalismo presupuestal en México: Un análisis de los programas presupuestarios del PEF 2010-2015. Gestión y Análisis de Políticas Públicas, (16).
Torres-Sánchez, L. E., Rojas-Martínez, R., Escamilla-Núñez, C., de la Vara-Salazar, E., y Lazcano-Ponce, E. (2014). Tendencias en la mortalidad por cáncer en México de 1980 a 2011. salud pública de méxico, 56(5):473-491. 